\title{
Intercomparison in spatial distributions and temporal trends derived from multi-source satellite aerosol products
}

\section{Jing Wei et al.}

Correspondence to: Yiran Peng (pyiran@ mail.tsinghua.edu.cn)

The copyright of individual parts of the supplement might differ from the CC BY 4.0 License. 
Table S1. Comparison between satellite-derived $\left(\mathrm{AOD}_{\mathrm{S}}\right)$ and ground-based monthly $\mathrm{AOD}_{\mathrm{A}}$ values during 2006-2010 over land and ocean

\begin{tabular}{cccccc|cccccc}
\hline Products & \multicolumn{3}{c}{ Land } & \multicolumn{5}{c}{ Ocean } \\
\hline Metrics & $\mathrm{N}$ & $\mathrm{R}$ & MAE & RMSE & $\mathrm{RMB}$ & $\mathrm{N}$ & $\mathrm{R}$ & MAE & RMSE & RMB \\
\cline { 2 - 11 } AATSR-ADV & 6979 & 0.734 & 0.086 & 0.153 & 0.868 & 959 & 0.712 & 0.068 & 0.100 & 1.149 \\
AATSR-EN & 7739 & 0.745 & 0.082 & 0.140 & 0.941 & 1023 & 0.711 & 0.061 & 0.093 & 1.063 \\
AATSR-ORAC & 8401 & 0.713 & 0.081 & 0.143 & 0.896 & 1066 & 0.696 & 0.069 & 0.100 & 1.204 \\
AATSR-SU & 7503 & 0.766 & 0.081 & 0.140 & 0.997 & 1026 & 0.693 & 0.058 & 0.098 & 0.988 \\
AVHRR & 7331 & 0.743 & 0.082 & 0.152 & 0.970 & 1051 & 0.783 & 0.047 & 0.078 & 1.004 \\
MISR & 7464 & 0.795 & 0.074 & 0.128 & 0.869 & 954 & 0.587 & 0.070 & 0.118 & 0.977 \\
MOD08 & 8108 & 0.875 & 0.074 & 0.113 & 1.162 & 1088 & 0.814 & 0.069 & 0.093 & 1.309 \\
MYD08 & 7945 & 0.870 & 0.068 & 0.110 & 1.090 & 1088 & 0.812 & 0.056 & 0.082 & 1.191 \\
POLDER & 7956 & 0.733 & 0.108 & 0.162 & 1.292 & 1027 & 0.694 & 0.071 & 0.110 & 1.247 \\
SeaWiFS & 4516 & 0.819 & 0.072 & 0.117 & 0.920 & 775 & 0.746 & 0.057 & 0.088 & 1.053 \\
\hline
\end{tabular}


Table S2. Statistics of continent-scale performance between monthly AODs and $\mathrm{AOD}_{\mathrm{A}}$ from 2006 to 2010

\begin{tabular}{|c|c|c|c|c|c|c|c|c|c|c|}
\hline $\mathbf{N}$ & $\begin{array}{l}\text { AATSR- } \\
\text { ADV }\end{array}$ & $\begin{array}{l}\text { AATSR- } \\
\text { EN }\end{array}$ & $\begin{array}{l}\text { AATSR- } \\
\text { ORAC }\end{array}$ & $\begin{array}{l}\text { AATSR- } \\
\text { SU }\end{array}$ & AVHRR & MISR & MOD08 & MYD08 & POLDER & SeaWiFS \\
\hline ENAM & 1013 & 1071 & 1276 & 1034 & 1137 & 1083 & 1191 & 1167 & 1143 & 672 \\
\hline WNAM & 1026 & 1089 & 1231 & 1072 & 1010 & 1116 & 1150 & 1127 & 1103 & 599 \\
\hline SAM & 504 & 551 & 542 & 562 & 469 & 509 & 569 & 553 & 568 & 304 \\
\hline EUR & 1856 & 1945 & 2163 & 1804 & 1960 & 1879 & 1972 & 1884 & 1954 & 1165 \\
\hline AFR & 716 & 869 & 878 & 865 & 769 & 832 & 878 & 879 & 874 & 567 \\
\hline ME & 115 & 286 & 286 & 285 & 213 & 286 & 286 & 286 & 286 & 248 \\
\hline SAA & 303 & 312 & 302 & 313 & 277 & 268 & 318 & 316 & 315 & 183 \\
\hline EAA & 696 & 726 & 805 & 689 & 767 & 703 & 804 & 805 & 812 & 341 \\
\hline SEA & 241 & 291 & 257 & 277 & 212 & 204 & 314 & 320 & 305 & 78 \\
\hline OCE & 244 & 307 & 308 & 313 & 235 & 304 & 318 & 310 & 312 & 223 \\
\hline \multicolumn{11}{|l|}{ MAE } \\
\hline ENAM & 0.049 & 0.047 & 0.056 & 0.045 & 0.044 & 0.048 & 0.056 & 0.047 & 0.133 & 0.047 \\
\hline WNAM & 0.046 & 0.057 & 0.048 & 0.046 & 0.053 & 0.046 & 0.056 & 0.051 & 0.098 & 0.051 \\
\hline SAM & 0.085 & 0.078 & 0.086 & 0.097 & 0.075 & 0.066 & 0.056 & 0.057 & 0.111 & 0.087 \\
\hline EUR & 0.049 & 0.046 & 0.043 & 0.044 & 0.046 & 0.048 & 0.056 & 0.045 & 0.074 & 0.043 \\
\hline AFR & 0.142 & 0.110 & 0.135 & 0.112 & 0.143 & 0.091 & 0.086 & 0.085 & 0.113 & 0.091 \\
\hline $\mathrm{ME}$ & 0.101 & 0.095 & 0.099 & 0.113 & 0.200 & 0.075 & 0.094 & 0.080 & 0.091 & 0.094 \\
\hline SAA & 0.200 & 0.183 & 0.170 & 0.173 & 0.129 & 0.148 & 0.155 & 0.136 & 0.145 & 0.174 \\
\hline EAA & 0.182 & 0.205 & 0.187 & 0.184 & 0.164 & 0.186 & 0.136 & 0.131 & 0.152 & 0.149 \\
\hline SEA & 0.150 & 0.120 & 0.123 & 0.114 & 0.137 & 0.127 & 0.113 & 0.115 & 0.158 & 0.130 \\
\hline OCE & 0.055 & 0.043 & 0.040 & 0.069 & 0.042 & 0.038 & 0.041 & 0.041 & 0.057 & 0.045 \\
\hline \multicolumn{11}{|l|}{ RMSE } \\
\hline ENAM & 0.071 & 0.066 & 0.078 & 0.065 & 0.064 & 0.072 & 0.078 & 0.067 & 0.189 & 0.069 \\
\hline WNAM & 0.069 & 0.089 & 0.079 & 0.076 & 0.072 & 0.068 & 0.076 & 0.073 & 0.129 & 0.076 \\
\hline SAM & 0.151 & 0.147 & 0.165 & 0.153 & 0.115 & 0.135 & 0.091 & 0.091 & 0.169 & 0.139 \\
\hline EUR & 0.068 & 0.065 & 0.062 & 0.066 & 0.070 & 0.071 & 0.075 & 0.062 & 0.117 & 0.059 \\
\hline AFR & 0.239 & 0.162 & 0.198 & 0.171 & 0.264 & 0.138 & 0.123 & 0.125 & 0.175 & 0.137 \\
\hline ME & 0.193 & 0.127 & 0.154 & 0.153 & 0.364 & 0.100 & 0.110 & 0.097 & 0.121 & 0.137 \\
\hline SAA & 0.267 & 0.236 & 0.226 & 0.226 & 0.166 & 0.191 & 0.212 & 0.187 & 0.198 & 0.226 \\
\hline EAA & 0.253 & 0.289 & 0.278 & 0.274 & 0.235 & 0.268 & 0.196 & 0.201 & 0.212 & 0.209 \\
\hline SEA & 0.269 & 0.180 & 0.191 & 0.180 & 0.202 & 0.191 & 0.156 & 0.163 & 0.225 & 0.184 \\
\hline OCE & 0.075 & 0.058 & 0.050 & 0.109 & 0.056 & 0.054 & 0.055 & 0.055 & 0.080 & 0.063 \\
\hline \multicolumn{11}{|l|}{ RMB } \\
\hline ENAM & 0.995 & 1.066 & 1.261 & 1.046 & 0.930 & 0.920 & 1.302 & 1.159 & 1.870 & 0.937 \\
\hline WNAM & 0.786 & 1.162 & 1.100 & 1.039 & 1.230 & 1.145 & 1.325 & 1.227 & 1.918 & 1.044 \\
\hline SAM & 0.992 & 0.902 & 0.790 & 1.218 & 0.998 & 0.851 & 0.941 & 0.915 & 1.467 & 0.877 \\
\hline EUR & 0.833 & 0.942 & 1.044 & 0.920 & 0.928 & 0.887 & 1.243 & 1.097 & 1.381 & 0.934 \\
\hline AFR & 0.846 & 0.985 & 0.706 & 1.103 & 1.062 & 0.893 & 1.032 & 1.000 & 1.084 & 0.971 \\
\hline ME & 0.846 & 1.206 & 0.862 & 1.243 & 1.329 & 1.005 & 1.271 & 1.210 & 1.024 & 1.120 \\
\hline SAA & 0.849 & 0.956 & 0.783 & 1.002 & 0.948 & 0.820 & 1.183 & 1.111 & 1.017 & 0.760 \\
\hline EAA & 0.793 & 0.705 & 0.723 & 0.765 & 0.824 & 0.658 & 1.113 & 1.098 & 1.068 & 0.755 \\
\hline SEA & 0.938 & 0.829 & 0.871 & 0.908 & 0.893 & 0.830 & 1.105 & 1.035 & 1.224 & 0.883 \\
\hline OCE & 1.457 & 1.405 & 1.307 & 1.776 & 1.148 & 1.252 & 1.188 & 1.151 & 1.548 & 1.395 \\
\hline
\end{tabular}


Table S3. Statistics of missing monthly data records for the eleven satellite-derived global aerosol products

\begin{tabular}{lll}
\hline Product & Count & Missing data records \\
\hline AATSR-ADV & 1 & 200706 \\
AATSR-EN & 0 & - \\
AATSR-ORAC & 6 & $200301 ; 200302 ; 200303 ; 200410 ; 200411 ; 201111$ \\
AATSR-SU & 0 & - \\
AVHRR & 1 & 200902 \\
MISR & 0 & - \\
MOD08 & 0 & - \\
MYD08 & 0 & - \\
POLDER & 0 & - \\
SeaWiFS & 7 & $200801 ; 200802 ; 200803 ; 200807 ; 200905 ; 200908 ; 200909$ \\
VIIRS & 0 & - \\
\hline
\end{tabular}




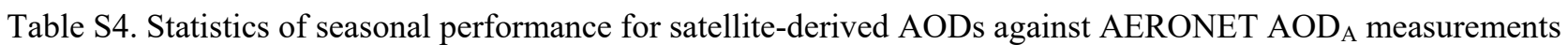
from 2006 to 2010

\begin{tabular}{|c|c|c|c|c|c|c|c|c|c|c|}
\hline DJF & $\begin{array}{l}\text { AATSR- } \\
\text { ADV }\end{array}$ & $\begin{array}{l}\text { AATSR- } \\
\text { EN }\end{array}$ & $\begin{array}{l}\text { AATSR- } \\
\text { ORAC }\end{array}$ & $\begin{array}{l}\text { AATSR- } \\
\text { SU }\end{array}$ & AVHRR & MISR & MOD08 & MYD08 & POLDER & SeaWiFS \\
\hline $\mathrm{N}$ & 735 & 811 & 995 & 787 & 885 & 919 & 911 & 867 & 955 & 639 \\
\hline MAE & 0.079 & 0.075 & 0.063 & 0.063 & 0.066 & 0.065 & 0.061 & 0.061 & 0.109 & 0.067 \\
\hline RMSE & 0.135 & 0.124 & 0.106 & 0.109 & 0.107 & 0.111 & 0.097 & 0.102 & 0.147 & 0.109 \\
\hline RMB & 0.845 & 0.919 & 0.952 & 0.966 & 1.026 & 0.815 & 1.105 & 1.051 & 1.500 & 0.867 \\
\hline МАM & $\begin{array}{l}\text { AATSR- } \\
\text { ADV }\end{array}$ & $\begin{array}{l}\text { AATSR- } \\
\text { EN }\end{array}$ & $\begin{array}{l}\text { AATSR- } \\
\text { ORAC }\end{array}$ & $\begin{array}{l}\text { AATSR- } \\
\text { SU }\end{array}$ & AVHRR & MISR & MOD08 & MYD08 & POLDER & SeaWiFS \\
\hline $\mathrm{N}$ & 909 & 956 & 971 & 952 & 920 & 946 & 965 & 959 & 923 & 791 \\
\hline MAE & 0.079 & 0.072 & 0.071 & 0.071 & 0.070 & 0.066 & 0.074 & 0.063 & 0.108 & 0.073 \\
\hline RMSE & 0.135 & 0.114 & 0.113 & 0.110 & 0.123 & 0.102 & 0.105 & 0.095 & 0.149 & 0.120 \\
\hline RMB & 0.906 & 0.950 & 0.929 & 1.022 & 0.979 & 0.920 & 1.206 & 1.118 & 1.309 & 0.994 \\
\hline JJA & $\begin{array}{l}\text { AATSR- } \\
\text { ADV }\end{array}$ & $\begin{array}{l}\text { AATSR- } \\
\text { EN }\end{array}$ & $\begin{array}{l}\text { AATSR- } \\
\text { ORAC }\end{array}$ & $\begin{array}{l}\text { AATSR- } \\
\text { SU }\end{array}$ & AVHRR & MISR & MOD08 & MYD08 & POLDER & SeaWiFS \\
\hline $\mathrm{N}$ & 971 & 1009 & 1003 & 1009 & 957 & 977 & 1012 & 1011 & 944 & 741 \\
\hline MAE & 0.081 & 0.078 & 0.095 & 0.077 & 0.087 & 0.076 & 0.085 & 0.074 & 0.103 & 0.078 \\
\hline RMSE & 0.137 & 0.143 & 0.164 & 0.132 & 0.193 & 0.139 & 0.121 & 0.114 & 0.153 & 0.137 \\
\hline RMB & 0.900 & 0.939 & 0.863 & 0.972 & 0.943 & 0.894 & 1.213 & 1.144 & 1.177 & 0.895 \\
\hline SON & $\begin{array}{l}\text { AATSR- } \\
\text { ADV }\end{array}$ & $\begin{array}{l}\text { AATSR- } \\
\text { EN }\end{array}$ & $\begin{array}{l}\text { AATSR- } \\
\text { ORAC }\end{array}$ & $\begin{array}{l}\text { AATSR- } \\
\text { SU } \\
\end{array}$ & AVHRR & MISR & MOD08 & MYD08 & POLDER & SeaWiFS \\
\hline $\mathrm{N}$ & 951 & 999 & 1006 & 987 & 964 & 972 & 995 & 993 & 949 & 739 \\
\hline MAE & 0.07 & 0.066 & 0.062 & 0.060 & 0.061 & 0.060 & 0.060 & 0.053 & 0.081 & 0.067 \\
\hline RMSE & 0.12 & 0.114 & 0.105 & 0.106 & 0.105 & 0.108 & 0.097 & 0.094 & 0.112 & 0.118 \\
\hline RMB & 0.895 & 0.945 & 0.976 & 0.979 & 0.941 & 0.859 & 1.130 & 1.039 & 1.292 & 0.928 \\
\hline
\end{tabular}



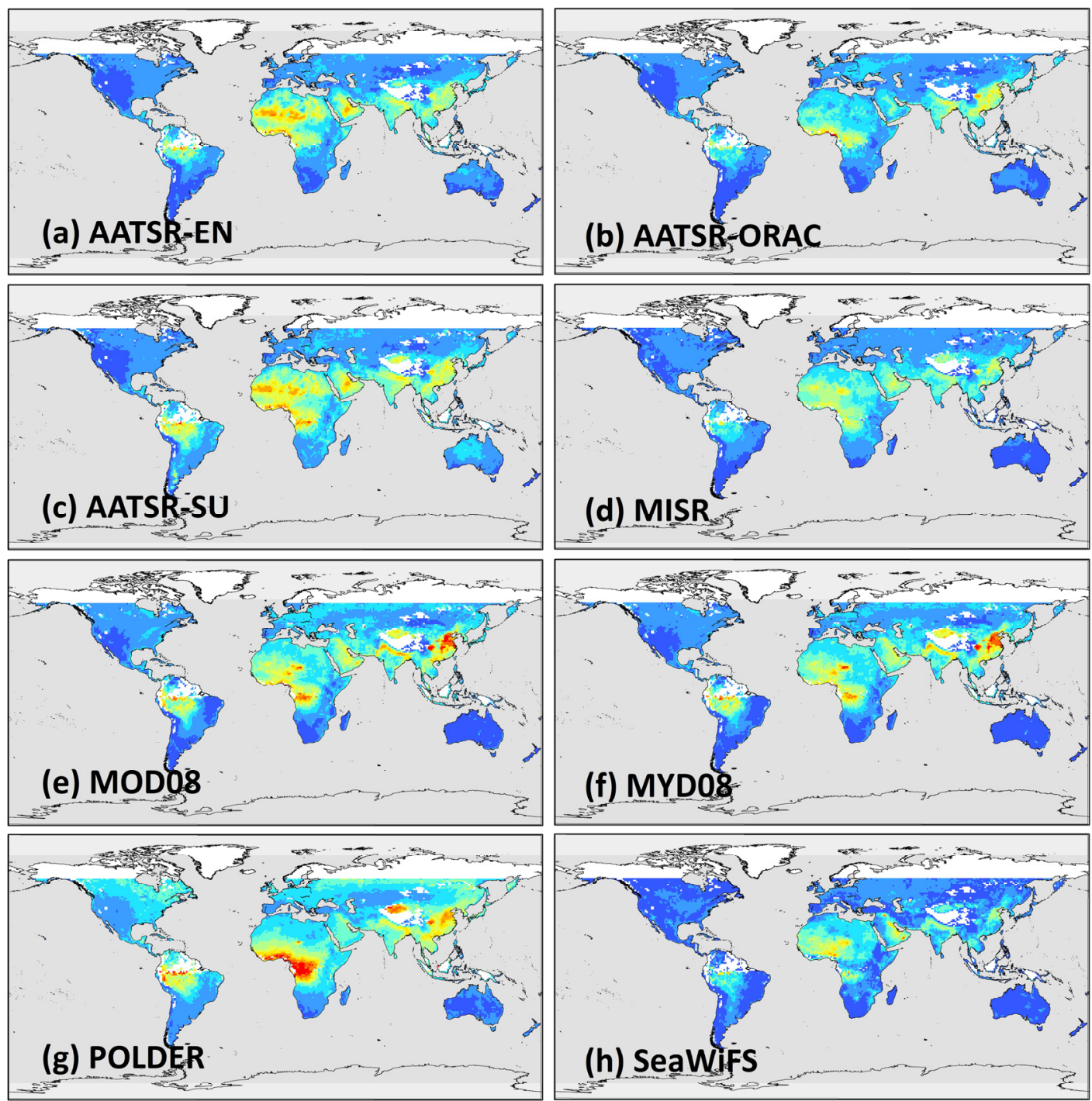
0.1
0.3
0.5
0.7
0.9

Figure S1. Satellite-derived global annual averaged AODs maps at $550 \mathrm{~nm}$ from 2006 to 2010 with common points in all datasets over land (exclude AATSR-ADV and AVHRR due to lack of observations over Sahara and the Middle East) 

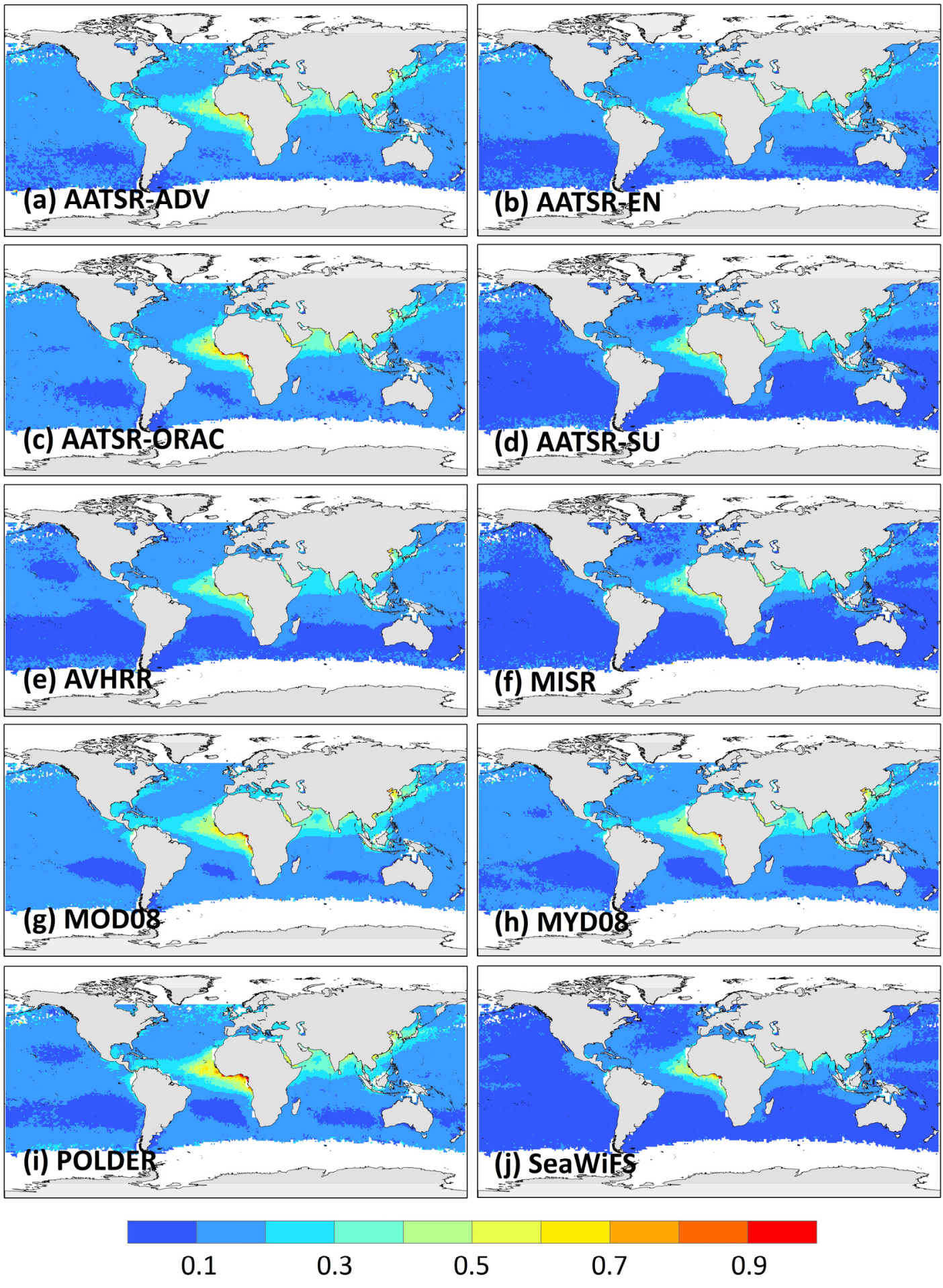

0.7

0.9

Figure S2. Satellite-derived global annual averaged AODs maps at $550 \mathrm{~nm}$ from 2006 to 2010 with common points in all datasets over ocean 

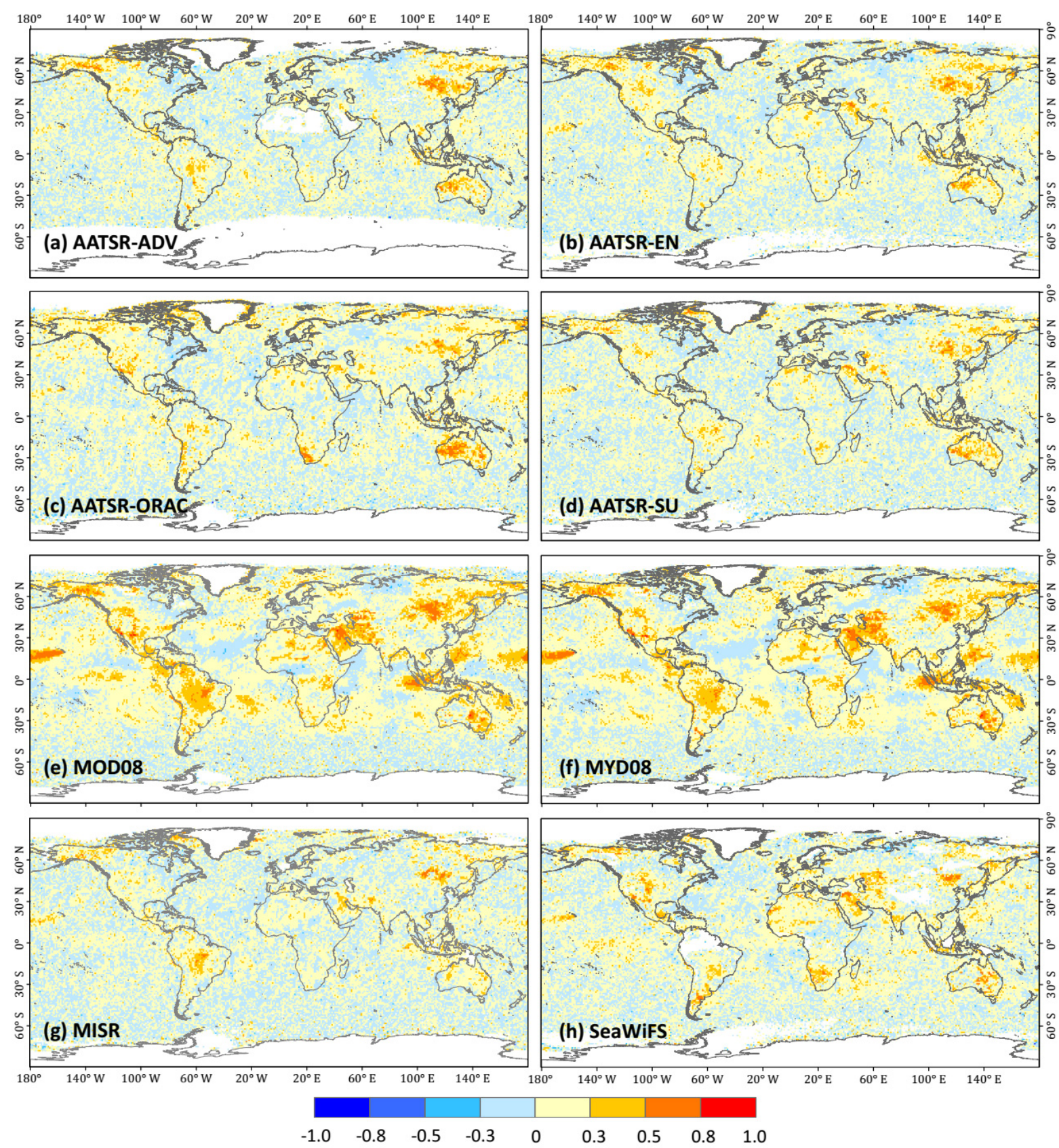

Figure S3. Spatial distribution of autocorrelation coefficient with a lag of one month based on de-seasonalized monthly $\mathrm{AOD}_{\mathrm{S}}$ anomalies at $550 \mathrm{~nm}$ from 2003 to 2010. 

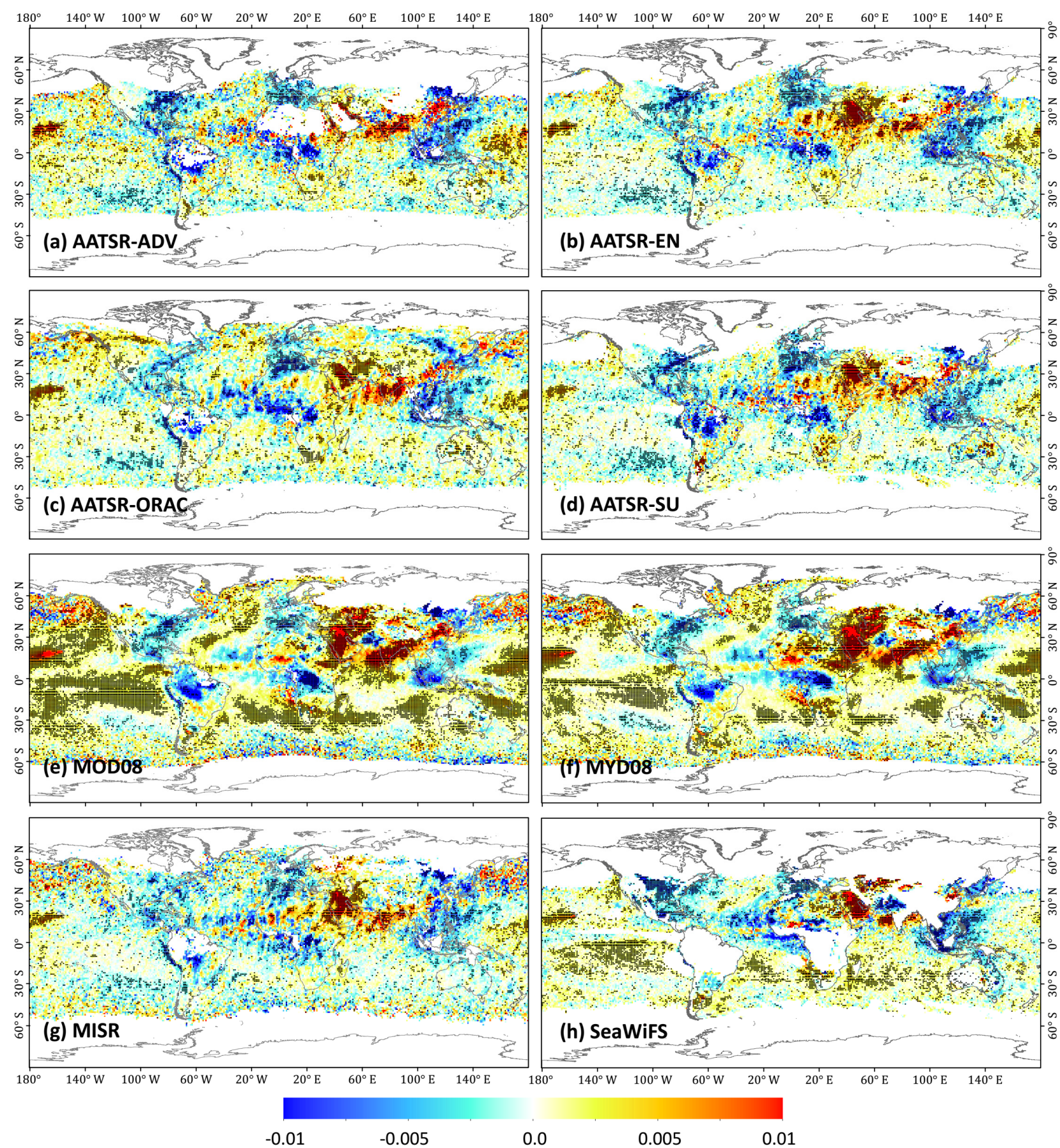

Figure S4. Linear trend based upon de-seasonalized monthly anomaly of AOD for the period 2003-2010. Units are AOD $\mathrm{yr}^{-1}$. Black dots indicate that trend is significant at the $95 \%$ confidence level $(\mathrm{p}<0.05)$ without the FDR correction. 